\title{
Performance Improvement Of Cellular \\ Systems Using MIMO-OFDM
}

\author{
W. M. Abdul-Latef \\ College of Electronics \\ Engineering \\ University of Mosul
}

\author{
S. A. Mawjoud \\ College of Engineering \\ University of Mosul
}

\begin{abstract}
The combination of multiple-input multiple-output (MIMO) system with OFDM techniques is regarded as a promising solution for enhancing the data rates of next-generation wireless communication systems.

SDM is implemented to transmitted different signals simultaneously at the same carrier frequency and to detect the transmitted signals using many techniques such as zero frequency, minimum mean square error with and without decision feedback and maximum likelihood decoding.

Furthermore, orthogonal frequency division multiplexing technique included in the simulation process using the parameters of the IEEE 802.11a standard. The fading channel is modeled by exponentially decayed Rayleigh fading channel which is based on the indoor WLAN, Least Square (LS) method is used to estimate the channel for each subcarrier.
\end{abstract}

Keyword: WCDMA, MIMO-OFDM.

تحسين أداء أنظمة الاتصالات الخلوية باستخدام

MIMO-OFDM
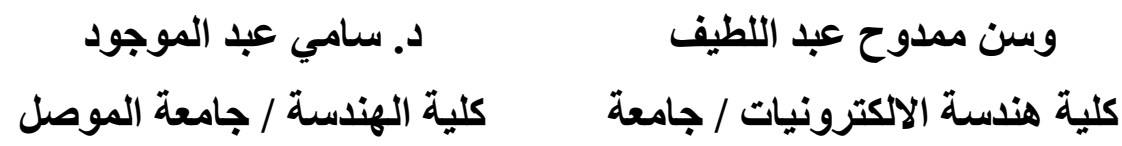


\section{الموصل}

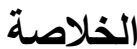

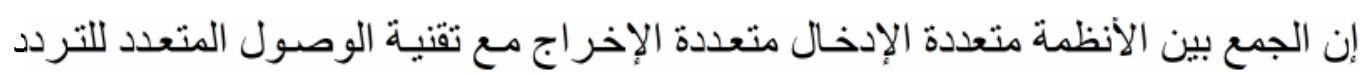

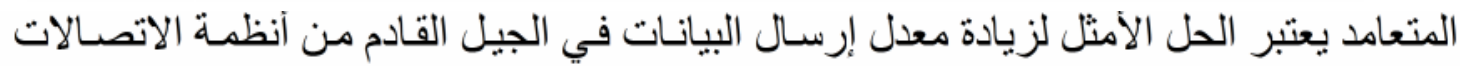

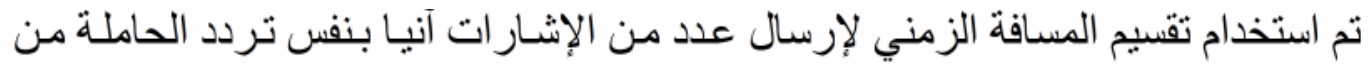

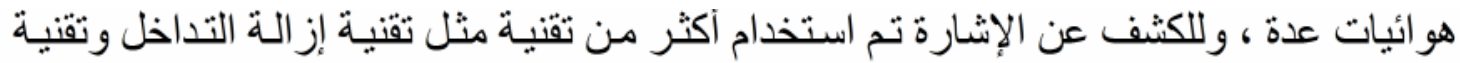
معدل مربع الخطأ الأقل مع التغذية العكسية وبدونها فضلا عن تقنية الكثف بأكبر احتمالية. بعد ذلك أضيفت تقنية الوصول المتعدد للتردد المتعامد إلى محاكاة النظام واستخدمت

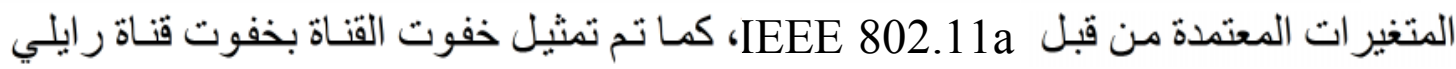

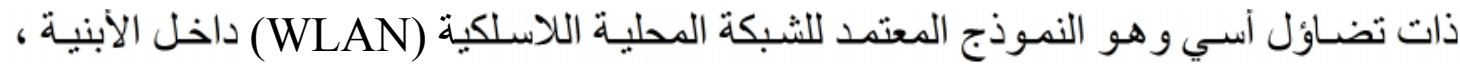
ولتخمين القتاة لكل تردد من ترددات الحاملـة الفر عية استخدمت طريقة مخمن التربيع الأقل

Received 23 Sep. 2007

Accepted 1 Nov. 2007

\section{1- Introduction}

The main goals in developing next-generation wireless communication system are to increase the link throughput (bit rate), provide high quality of service and efficient use of bandwidth. Important improvements in wireless system can be achieved when multiple antennas are applied at both the transmitting and receiving sides, especially in rich scattering environments [1].

Multiple input multiple output can generally be defined as a MIMO system in which $\mathrm{N}_{t}$ signals are transmitted by $\mathrm{N}_{t}$ antennas at the same time using the same bandwidth and effective processing at the receiver side based on the $\mathrm{N}_{\mathrm{r}}$ received signals by $\mathrm{N}_{\mathrm{r}}$ different antennas, is able to distinguish the different transmitted signals [2]. 
MIMO systems differs from traditional multiple access techniques, some of these differences are worth pointing out.

First, unlike code division multiple accesses (CDMA) or other spread spectrum multiple access techniques, the total channel bandwidth utilized in a MIMO system is only a small fraction in excess of the symbol rate, i.e. similar to the excess bandwidth required by a conventional Quadrature Amplitude Modulation (QAM) system. Second, unlike FDMA, each transmitted signal occupies the entire system bandwidth. Finally, unlike TDMA, the entire system bandwidth is used simultaneously by all of the transmitters all of the time [3].

MIMO techniques can basically be split into two groups: space time coding (STC) [4] and space division multiplexing (SDM) [1] [3]. STC increases the performance of the communication system by coding over the different transmitter branches; whereas SDM achieves a higher throughput by transmitting independent data streams on different transmit branches simultaneously and at the same carrier frequency [1]. Since increasing the bit rates is one of the goals, focusing on SDM algorithms is done in this paper.

A potential application of the MIMO principle is the next-generation wireless local area network (WLAN). The current WLAN standers IEEE 802.11a [5] are based on orthogonal frequency division multiplexing (OFDM) [6] [7]. A potential high data rate extension of these standards could be based on MIMO. This leads to the promising combination of the data rate enhancement of SDM with the relatively high spectralefficiency and the robustness against frequency selective fading channel. Advantage of wireless LAN systems is that they are mainly deployed in indoor environments. These environments are typically characterized by a richly scattered multipath [8] ... [10].

\section{2-MIMO Model}

In this section, a signal model for the multi antenna link will be stated in which the communication channel bandwidth is assumed to be so narrow than the channel can be treated as flat with frequency (i.e. flat fading) [11][12]. 
A communication system comprising $\mathrm{N}_{t}$ transmit (Tx) and $\mathrm{Nr}$ receive

$(\mathrm{Rx})$ antennas will be considered .It is assumed to operate in a Rayleigh flat fading environment and exploits the spatial dimension by using Space Division Multiplexing (SDM), see Fig. (1) where MAPU stands for Multi Antenna Processing Unit). At discrete times, the transmitter sends an $\mathrm{N}_{t}$ dimensional complex signal vector $\mathrm{x}$. The receiver records an $\mathrm{N}_{\mathrm{r}}$ dimensional complex vector $\mathrm{r}$. The following signal model describes the relation between $\mathrm{x}$ and $\mathrm{r}[12]$ :

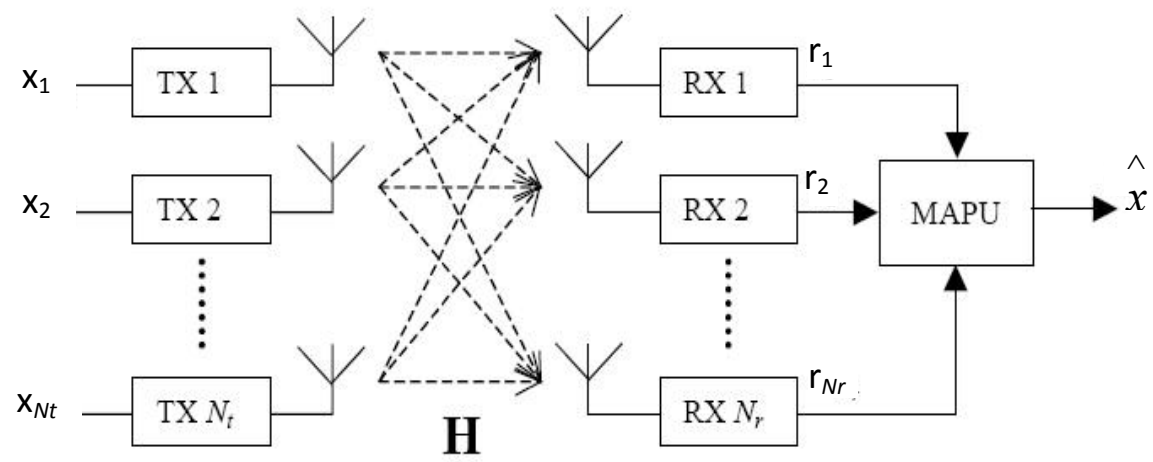

Fig. (1) The physical model of a system with Space Division Multiplexing (SDM) 
Where $H$ is an $\mathrm{N}_{\mathrm{r}} \times \mathrm{N}_{\mathrm{t}}$ complex propagation matrix that is constant with respect to the symbol time and assumed to be known at the receiver (e.g. via transmitting training sequences).

Since it is assumed that the system operates in a Rayleigh flat fading environment, it can be said that $H$ has independent and identically distributed (i.i.d.), zero mean, circularly symmetric, complex Gaussian entries with unit variance (the variance of each entry is $\sigma_{c}^{2}=1$ ).

The $\mathrm{N}_{\mathrm{r}}$ dimensional vector $\mathrm{v}$ represents zero mean, complexes Additive White Gaussian Noise (AWGN) with covariance matrix.

$E\left[v v^{*}\right]=\sigma_{v}^{2} I_{N_{r}}$

Where $*$ denotes the conjugate transpose of a matrix. The matrix $I$ with subscript $\mathrm{N}_{\mathrm{r}}$ represents the identity matrix with dimension $\mathrm{N}_{\mathrm{r}}$. The vector $\mathrm{x}$ is assumed to have Zero mean uncorrelated random variables with variance $\sigma_{x}^{2}$. The total power of $\mathrm{x}$ is assumed to be $\mathrm{P}$ (independent of the number of transmit antennas). Thus, the covariance matrix of $\mathrm{x}$ is

$$
E\left[x x^{*}\right]=\sigma_{x}^{2} I_{N_{t}}=\frac{P}{N_{t}} I_{N_{t}}
$$

Furthermore, the vectors $\mathrm{x}$ and $\mathrm{v}$ are assumed to be independent ( $\left.E\left[x v^{*}\right]=0\right)$. Now, the expected signal to noise ratio (SNR) per receiving antenna, i.e. the SNR for each component of $r$, can be found and is given as

$$
\rho=\frac{E_{s}}{N_{o}}=\frac{N_{t} \sigma_{x}^{2} \sigma_{c}^{2}}{\sigma_{v}^{2}}=\frac{P}{\sigma_{v}^{2}}
$$


Where $E_{s}$ is the signal power per receive antenna and $N_{o}$ is the noise power per receive antenna [13].

Five different algorithms have been considered in this paper for MIMO detection, providing us with a good range of complexity and performance.

\section{1- Zero Forcing (ZF) Receivers}

The first decoding technique forms the basis of the following decoding algorithms to be described in this paper (except for Maximum Likelihood Decoding). In this technique, each substream in turn is considered to be the desired signal, and the remaining data streams are considered as "interferers". Nulling of the interferers is performed by linearly weighting the received signals so that all interfering terms are cancelled such that

$D \cdot H=I$

Where $\mathrm{D}$ is a matrix that represent the linear processing in the receiver and $I$ is the identity matrix. So, by forcing the "interferers" to zero, each desired element of $\mathrm{x}$ can be estimated. If $\mathrm{H}$ is not a square matrix, D equals the pseudo-inverse of $\mathrm{H}$ :

$D=H^{+}=\left(H^{*} H\right)^{-1} H^{*}$

Where + represent the pseudo-inverse, the estimates of $\mathrm{x}$ (given by $\mathrm{x}_{\mathrm{est}}$ ) can be found by: [3]

$x_{e s t}=D x$ 


\section{2- The Minimum Mean Square Error}

Another approach in estimation theory is the problem of estimating a random vector $\mathrm{x}$ on the basis of observations $\mathrm{r}$ is to choose a function $\mathrm{g}(\mathrm{r})$ which minimizes the Mean square error (MSE)

$\varepsilon^{2}=E\left[\left(x-x_{e s t}\right)^{*}\left(x-x_{e s t}\right)\right]=\left[(x-g(r))^{*}(x-g(r))\right]$

To minimize the Mean Square Error over D, the processing at the receiver must be equal to :

$D=\left(\alpha I+H^{*} H\right)^{-1} H^{*}, \alpha>0$

Where $\alpha$ is equal to $\sigma_{v}^{2} / \sigma_{x}^{2}=N_{t} / \rho$. From equation (9) it becomes clear that the ZF solution corresponds to an MMSE solution with $\alpha=0$ [13].

\section{3- Zero Forcing With Decision Feedback Decoding}

The linear nulling approach as described in section (2.1) is viable, superior performance is obtained if non-linear techniques are used. One can imagine that if somehow first the most reliable elements of $\mathrm{x}$ superior performance can be achieved. This is called symbol cancellation [1] and it exploits the timing synchronism inherent in the system model (the assumption of co-located transmitters makes this completely reasonable).

Furthermore, linear nulling (i.e. ZF) or MMSE is used to perform detection. In other words, symbol cancellation is based on the subtraction of interference from already detected components of $x$ from the receiver signal vector $r$. This results in a modified receiver in which, effectively fewer interferers are present. Because this principle is somewhat analogous to decision feedback equalization, it is called Decision Feedback Decoding (DFB). 


\section{4- Minimum Mean Square Error With Decision Feedback Decoding}

In order to perform Decision Feedback Decoding with Minimum Mean Square Error Decoding, the DFB algorithm of section 2.4 has to be adapted somehow, the covariance matrix of the estimation error $\left(\mathrm{x}-\mathrm{x}_{\mathrm{est}}\right)$ will be used to determine a good ordering of detection. For MMSE, this covariance matrix can be show to be:[10]

$$
\begin{aligned}
E\left[\left(x-x_{\text {est }}\right)\left(x-x_{\text {est }}\right)^{*}\right]= & E\left[(x-D(H x+v))(x-D(H x+v))^{*}\right\rfloor \\
& =\sigma_{v}^{2}\left(\alpha I+H^{*} H\right)^{-1} \cong \sigma_{v}^{2} P \quad \ldots .
\end{aligned}
$$

\section{5- Maximum Likelihood Decoding}

MLD is a method that compares the received signal with all possible transmitted signals and estimates $\mathrm{x}$ according to the Maximum Likelihood principle. Suppose a matrix $\mathrm{C}$ gives all possibilities in $\mathrm{x}$ that could occur (the dimensions of $\mathrm{C}$ are $\mathrm{N}_{\mathrm{t}} \times \mathrm{K}$ where $\mathrm{K}=Q^{N_{t}}$ and $Q$ represents the number of constellation points). Then, the receiver should store a matrix Y such that.

$\mathrm{Y}=\mathrm{H} . \mathrm{C}=\left[y_{1} \ldots \ldots \ldots y_{K}\right]$

At the receiver, the most likely transmitted signal is determined, as the one for which $\left\|x-y_{i}\right\|^{2}$ is minimal (with $1 \leq \mathrm{j} \leq \mathrm{K}$ ), i.e., the signal $\mathrm{x}_{\mathrm{j}}$ that corresponds with the vector $y_{i}$ which is closest to the received vector is said to be the most likely signal to be transmitted. Thus, $\hat{x}$ is chosen to be the $\mathrm{j}$-th column of matrix $\mathrm{C}$. This can be rewritten to the following formula where $x_{m l}$ represents the Maximum likelihood detection of the transmitted signal $\mathrm{x}$ : [13] 


$$
\hat{x}=x_{m l}=\underset{x_{j} \in\left\{x_{1} \ldots \ldots . x_{K}\right\}}{\arg \min }\left\|r-H x_{j}\right\|^{2}
$$

\section{3- Simulation Result}

After discussing of the detection techniques in MIMO, using Matlab 7, a comparison has been drawn concerning the performance of the five mentioned techniques as shown in Fig (2) with BPSK and Fig. (3) with QPSK, the number of transmitting and receiving antenna has been fixed to two antennas each.

From figures (2) and (3), it can be concluded that the method of MLD is the best for minimum bit error rate but this method needs large dimension matrix $\mathrm{Y}$ spatially by increasing the elements of QAM or

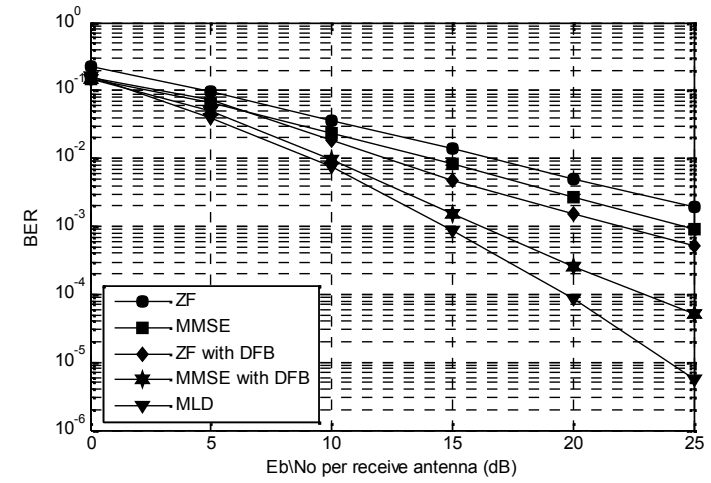

Fig [2]: BER verses mean $E_{b} / N_{o}$ per receiving Fig [3]: $B E R$ verses mean $E_{b} / N_{o}$ per receiving antenna for antenna configuration $\left(N_{t}, N_{r}\right)=$ antenna for antenna configuration $\left(N_{t}, N_{r}\right)=$ $(2,2)$, BPSK

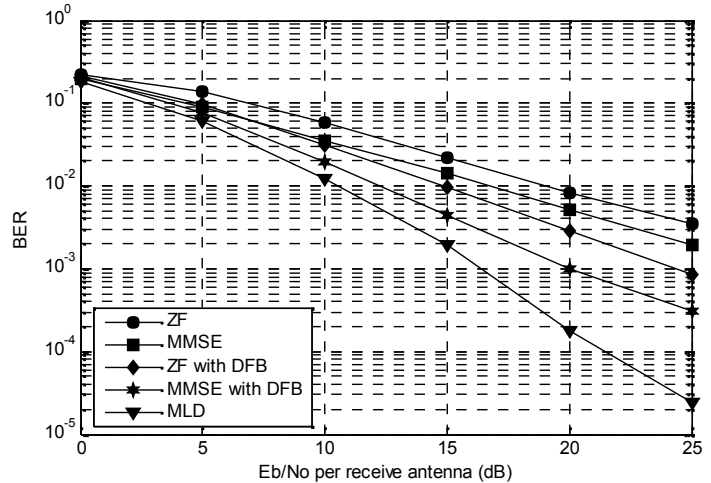

$(2,2)$, QPSK

increasing the number of transmitting antennas $N_{t}$, and this result in complication in the detection of the signal and increase the processing time.

Following the MLD method is the MMSE with DFB and then ZF with DFB. The important merits of these methods is the ability to deal with many transmitting signals with improve bit error rate, but the two above mention methods needs longer time for processing compare with MMSE, ZF methods. 
Next is the MMSE, ZF methods which are characterized by simplicity in execution but the BER is higher spatially when increasing the number of transmitting antennas $N_{t}$.

From figure (2) it can be observed that the results using MLD and MMSE with DFB near each other in the range between $0 \mathrm{~dB} \rightarrow 20 \mathrm{~dB}$, a bit error rate of $9 \times 10^{-3}$ was obtained in the case of MMSE with DFB at a signal to noise ratio of $20 \mathrm{~dB}$ and the same result under the same circumstances using MLD at $18 \mathrm{~dB}$, the difference in performance is clear after $20 \mathrm{~dB}$.

In figure (3) it can be observed that the increased difference in bit error rate in the two methods, which means that the MMSE with DFB is affected by the increased elements of modulation QAM more than MLD, knowing that the latter becomes more complex as the number of QAM is increased.

Therefore from the previous discussion we can conclude that detection using MMSE with DFB is the appropriate method compared with other methods from the number of signals in receiving side with decreasing bit error rate, also this method is less complex compared to detection method using MLD.

\section{4- OFDM System}

Figure 4 shows the block diagram of a generic OFDM system that has been implemented in matlab following the IEEE 802.11a standard. The system contains one transmitted and one received antennas where no source or channel coding is used.

The OFDM transmitter first generates equally probable bits for the mapper. The mapper maps the bits according to the selected modulation: binary phase shift keying (BPSK) or quadrature amplitude modulation (QAM). After the constellation points are inserted on the specified subcarriers. In the serial to parallel block, the serial input symbol - stream is converted to a parallel stream with width equal to the number of subcarriers. 


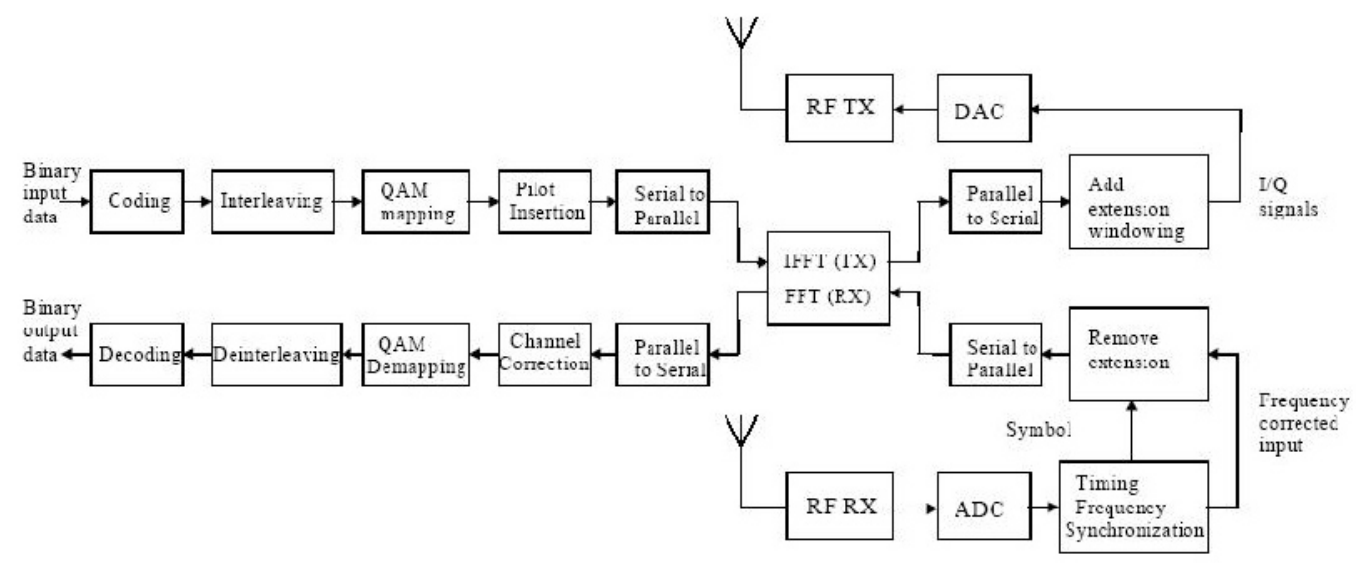

Fig (4): Block diagram of an OFDM transceiver

These parallel symbols are modulated on to the subcarriers by applying the Inverse Fast Fourier Transform (IFFT). After the IFFT block, the parallel output is converted back to serial. To make the system robust to multipath propagation, a cyclic prefix is added. After this step, the digital output signals are converted to analog signal. These analog signals are then up converted to the RF band, amplified and transmitted through an antenna.

The OFDM receiver basically performs the reverse operations of the transmitter, together with additional training tasks. First, the receiver has to estimate the channel using special training symbols in a preamble, and then it is able to excite the Fast Fourier Transform for every symbol to recover the QAM values of all subcarriers, the QAM values are then damped into binary values and can decode the information bits [5].

\section{5- MIMO-OFDM}

MIMO techniques are based on the assumption of a flat fading channel. However the use of OFDM modulation makes the flat fading hypothesis true for each OFDM sub-band, allowing exploitation of the MIMO approach for broadband wireless applications as well.

The transmitter is equipped with $\mathrm{N}_{t}$ antennas .The source information bits are mapped on the symbols of the constellation adopted for each OFDM subcarrier, the vectorial nature of the transmission introduced by demultiplexing $\left(1 \rightarrow \mathrm{N}_{\mathrm{t}}\right)$ these symbols. It maps the symbols on the $\mathrm{N}_{t}$ space channels, A serial to parallel converter for each space 
channel takes $\mathrm{n}$ of these symbols to form the input for the OFDM modulator. To avoid any inter symbol interference (ISI) due to the delay spread of the channel, a cyclic prefix is appended to each OFDM symbol.

The receiver is equipped with $\mathrm{N}_{\mathrm{r}}$ antennas (note that MMSE with DFB requires $N_{r} \geq N_{t}$ ). Each antenna receives a different noisy superposition of the faded versions of the $\mathrm{N}_{t}$ transmitted signals. If the antennas are sufficiently spatially separated (more than $\lambda / 2$ ) and there is sufficiently rich scattering, the transmitted signals arriving at different receive antennas undergo independent fading. Moreover, if the channel response is known at the receiver MMSE with DFB algorithm is able to detect the $\mathrm{N}_{\mathrm{t}}$ transmitted signals.

Channel response can be estimated at the receiver using a training sequence. In so doing, the channel is assumed to remain fixed during a frame, but it randomly changes from frame to frame.

At each OFDM symbol time, the output of OFDM demodulator corresponding to the receiver antenna $\mathrm{N}_{\mathrm{r}}$ is a set of $\mathrm{n}$ signals $r_{n_{r}, n}$ one for each frequency channel

$$
r_{n_{r}, n}=\sum_{n_{t}=1}^{N_{t}} h_{n_{r}, n_{t}, n} \cdot x_{n_{t}, n}+v_{n_{r}, n}
$$

\section{Where}

$r_{n_{r}, n}:$ receive signal for $\mathrm{n}_{\mathrm{r}}$ receiver antenna at subcarrier frequency $\mathrm{n}$

$h_{n_{r}, n_{t}, n}$ : channel fading for $\mathrm{n}_{\mathrm{t}}$ transmit antenna and $\mathrm{n}_{\mathrm{r}}$ receive antenna at subcarrier

frequency $n$

$x_{n_{t}, n}:$ transmit signal for $\mathrm{n}_{\mathrm{t}}$ transmit antenna at subcarrier frequency

$v_{n_{r}, n}$ : Adaptive white Gaussian noise for $\mathrm{n}_{\mathrm{r}}$ receiver antenna at subcarrier $\mathrm{n}$

The $n_{r}$ outputs related to the multicarrier frequency $n$ are the inputs to the MMSE with DFB signal processor that detects the $\mathrm{N}_{t}$ different $\mathrm{M}$ - 
QAM symbols transmitted in this subcarrier frequency. There are $n$ MMSE with DFB signal processors, each operating in subcarrier frequency.

The symbols obtained the output of the $\mathrm{n}$ MMSE with DFB signal processors, are then serialized by a parallel to serial converter in order to complete traditional receiver processing [2][9].

\section{6- The Channel}

\section{a- Indoor Channel Model}

In this paper the channel model recommended by the IEEE $802.11 \mathrm{a}$ standard specification [14] is used. This channel model is referred to "Exponentially Decaying Rayleigh Fading Model" [13][15] as shown in (FIG 5a); this model represents a real world scenario in which the positions of the reflectors generate paths that are longer and longer

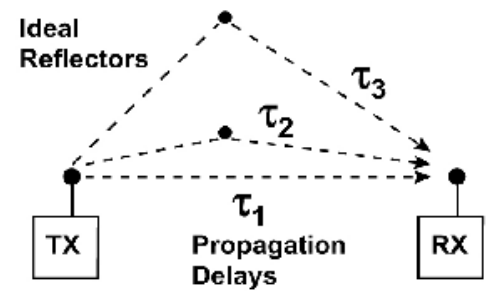

(a)

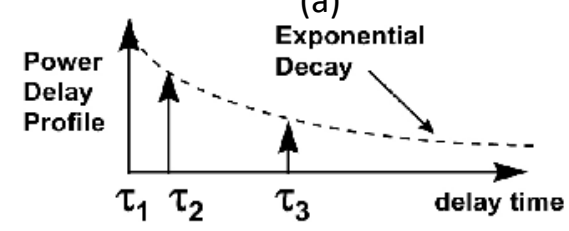

(b)

Fig (5): Exponentially decayed Power Delay Profile (DDP) 
The Power Delay Profile (PDP) for the exponentially-decayed Rayleigh fading channel is shown in Fig (5b). The power delay profile (PDP) measures the mean signal power relative to its dispersion across time. The mean power level establishes the variance of the corresponding Rayleigh components. The excellent features of exponentially decayed multipath is that on average the strongest paths arrive earliest in time since propagation delay is proportional to distance traveled. Radiated power drops off logarithmically with respect to distance [15].

\section{b- Channel Estimation}

Channel estimations for MIMO-OFDM systems can be obtained by transmitting a training sequence [15]...[17] from one antenna at a time while the remaining transmits antennas are idle.

A simple way of designing such training sequences is by sending a pilot symbol on subcarriers $\mathrm{N}$ of antenna $\mathrm{n}_{\mathrm{r}}$ and zeros on subcarriers for all other antennas as shown in (Fig 6).

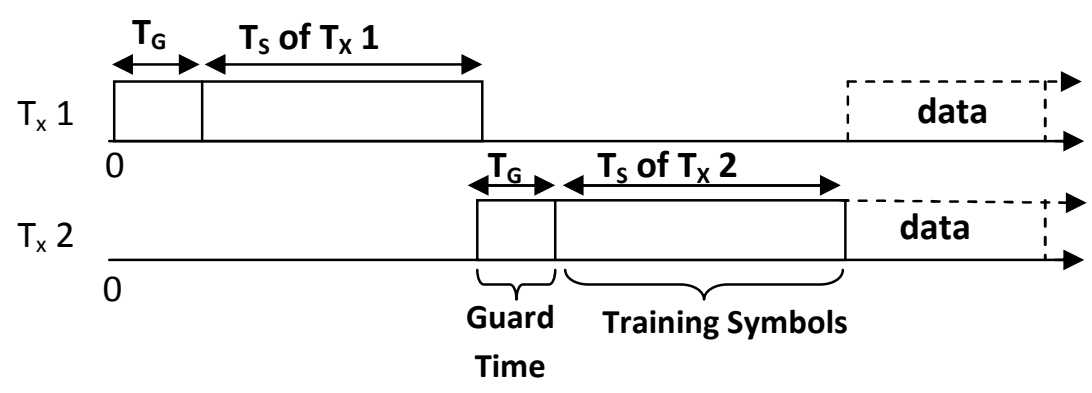

Fig (6): Training symbol in (MIMO-OFDM) system 
This type of channel estimation assumes slow fading channels, where the channel effect is not changed during one packet and this is a valid assumption for indoor WLAN system.

Considering a MIMO-OFDM system with $\mathrm{N}_{t}$ transmit, $\mathrm{N}_{\mathrm{r}}$ receive antennas, and $\mathrm{n}$ subcarriers see equ (13), with only one antenna is transmitting training sequence. Note that equ (13) can be reduce to $r_{n_{r}, n}=h_{n_{r}, n_{t}, n} \cdot x_{n_{t}, n}+v_{n_{r}, n}$

Then $h_{n_{r}, n_{t}, n}$ can be estimated by simply dividing $r_{n_{r}, n}$ by the known training symbol $x_{n_{t}, n}$ where

$h_{n_{r}, n_{t}, n}=\frac{r_{n_{r}, n}}{x_{n_{t}, n}}$

This method of estimation named (Least Square LS), and the important merits of this method is simplicity and of less complexity compared with other channel estimation methods, which is important in practice but it is more affected by high noise level [18].

\section{7- Simulation Results In MIMO-OFDM}

Simulation of MIMO-OFDM systems have been implemented using (matlab v7). The main goal of the simulation is to compare the performance when changing the number of transmitting and receiving antennas, in addition the changing of digital phase modulation method using BPSK\& QPSK.

The algorithm SDM is implemented using MMSE with DFB because this method is the most appropriate for the detection of the signal as mention before. Also the use of IEEE 802.11a for the implementation (OFDM), taking into consideration the following assumptions. 
1- There is synchronization between the transmitting and receiving sides (without using pilot carriers).

2- Coding has not been included in simulation.

3- The bandwidth is divided in to 64 subchannels, 48 of it for information; the OFDM symbol time used $3.2 \mu$ s in addition of $0.8 \mu$ s guard time, so that the overall time is $4 \mu \mathrm{s}$.

4- The average of 500 iteration since it was found that it gives acceptable result.

5- The estimation of channel $\mathrm{H}$ has been implemented using least square error estimator as mentioned in $6 b$.

6- The choice of channel time delay used is $50 \mathrm{~ns}$ as this number represent a case of many cases to represent the channel [19].

Figure (7) represent simulation of OFDM using BPSK with $\mathrm{N}_{t}$ $=\mathrm{Nr}=1$.the figure shows the difference of system performance when the channel matrix $\mathrm{H}$ is known at the receiving sides and when estimated using LS estimator it can be notice the difference is about $3 \mathrm{~dB}$.

The result obtain when $\mathrm{Nt}=\mathrm{Nr}=1$ and the knowledge of the channel in the receiving side the second curve in Figure (7) which agrees with a result obtain in [18]. Knowing that the variable used to represent (MIMOOFDM) system is not dealt with in previous papers.

The number of antennas is the most important factor affecting the system performance, while the number of antennas on the receiving side have changed.

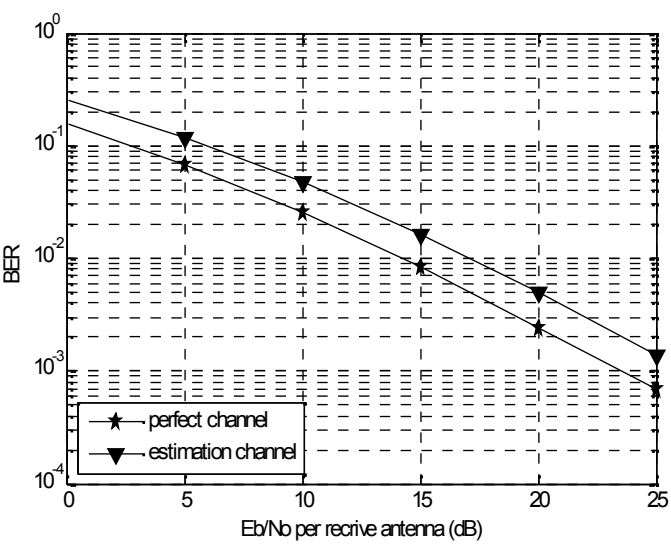

Fig (7): BER verses mean $E_{b} / N_{o}$ per receiving antenna for antenna configuration (N.. N.) = (1. 1). BPSK

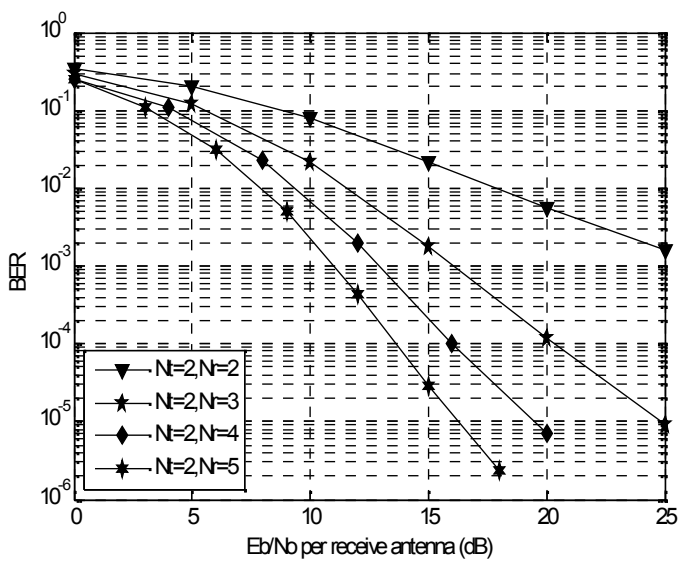

Fig (8): The effective of increasing $N_{r}$ in (MIMO-OFDM) system with $\mathrm{N}_{\mathrm{t}}=\mathbf{2}$ 
Figure (8) represents the simulation of MIMO-OFDM using $\mathrm{Nt}=2 \&$ varying the number of antennas at the receiving side $\mathrm{Nr}=2 \rightarrow 5$ using (BPSK), Figure (9) presents the above case but using QPSK, from the

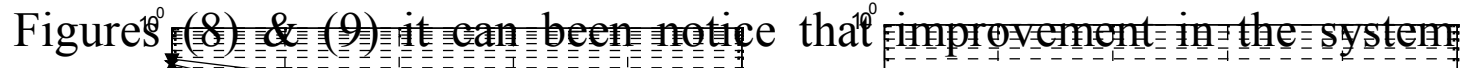

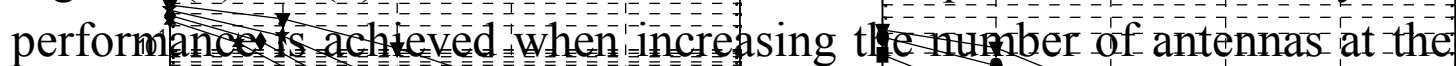
receiving side.

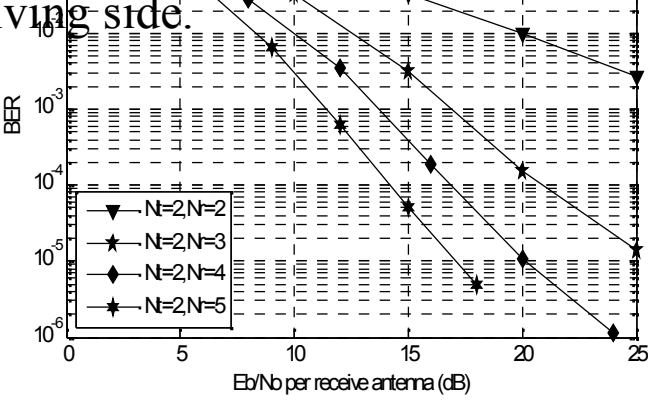

Fig (9): The effective of increasing $N_{r}$ in (MIMO-OFDM) system with $\mathrm{N}_{\mathrm{t}}=\mathbf{2}$

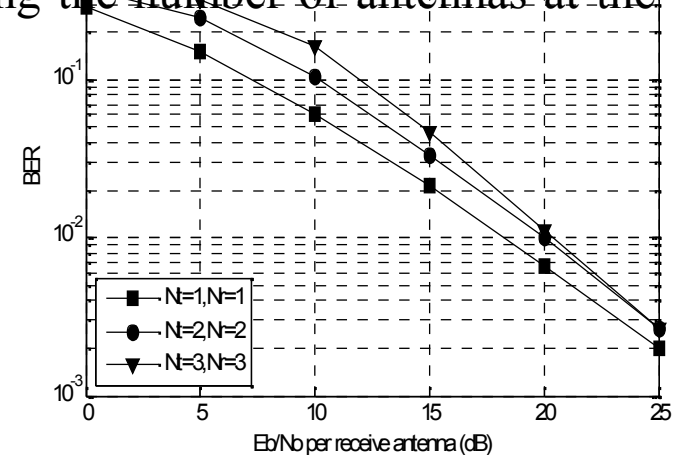

Fig (10): The effective of increasing $N_{t} \& N_{r}$ in (MIMO-OFDM) system with QPSK 
In the case that the number of antennas $\mathrm{Nt}=\mathrm{Nr}$ as show in Figure (10) it can be concluded that using MMSE with DFB techniques for the detection of the signal can be achieved with increase in the data rate without increase in bit error rate.

\section{8-Conclusions}

- The combination of MIMO and OFDM techniques comply with the requirement of future communication system (high data rate $>>2 \mathrm{M}$ bps) and efficient used of the frequency spectrum $(20 \mathrm{MHz})$ in addition of simple implementation of the circuit used in OFDM, the method of estimation (LS) used in this paper is considered the simplest which has considerable importance for practical purposes.

- Using the techniques of MMSE with DFB in MIMO-OFDM increase the data rate without considerable difference in bit error rate.

- The method of MLD is considered the best in performance and the most complex when increasing the number of transmitting antennas.

- The method of MMSE with DFB is the most suitable for the detection of the signal compared with other methods from the point of dealing with number of signals in the receiving side with less bit error rate, in addition to less complexity compare to MLD method.

\section{References :}


1- Zelst, A.V., Schenk, T.C.W., "Implementation of a MIMO OFDM Based Wireless LAN System", IEEE Translations on Signal Processing, Vol. 52, No. 2, February, 2004.

2- Giangaspero, L. and Agarossi, L., "Co - Channel Interference Cancellation Based on MIMO OFDM Systems", IEEE Wireless Communication, December, 2002.

3- Wolniansky, P.W., Foschini, G.J., Golden, G.D. and Valenzuela. R.A., "V - BLAST: An Architecture for Realizing Very High Data Rates Over the Risk - Scattering Wireless channel", 1998 URSI International symposium on Signals, Systems and Electronics, ISSSE 89, Pisa, 29 Sept. - 2 Oct., 1998, pp 295-300.

4- Alamouti, S.M., "A Simple Transmit Diversity Technique for Wireless Communications", IEEE Journal on Select Areas on Communications, Vol. 16, No. 8, October, 1998.

5- Prasad, N. and Prasad, A., "WLAN Systems and Wireless IP for Next Generation Communications", Universal Personal Communication, Boston, London, 2002.

6- Lawerey, E.P., "Adaptive Techniques for Multiuser OFDM", Ph.D Thesis, James Cook University, December, 2001.

7- Wang, T., Praokis, J.G. and Zeidler, J.R., "Techniques for Suppression of Intercarrier Interference in OFDM Systems", IEEE Communication Society / ECNC, 2005.

8- Xiao - Yun, H., Bao - Yu, Z., You - Yun, X. and Wen - Tao, S., "An Improved Channel Estimation with Multipath Search for MIMO OFDM Systems", Journal of Zhejiang University SCIENCE A, 2006, pp 149-155.

http://www.zju.edu.cn/jzus.

9- Whu, E.U., "MIMO - OFDM Systems for High Data Rate Wireless Networks", EE360 Advanced Wireless Networks, Stanford University, 2003.

10- Collados, M., Dolmans, G., Modonesi, I., schenk., T., Zelst, A.V., "Deliverable D 3.4: WP3 Overall Public Deliverable", Broadband Radio@ Hand, Philips - WP3 - PUB - 01 -WP3 overview, July, 2005 . 
11- Rappaport, T.S., "Wireless Communications Principles and Practice", Publishing House of Electronics Industry, 1996.

12-Correia, L.M., "Wireless Flexible Personalised Communications", Cost 259: European Co - operation in Mobile Radio Research, June, 2001.

13- Zelst, A.V., "Extending the Capacity of Next Generation Wireless LANs Using Space Division Multiplexing Combined with OFDM", MSc. Thesis, Technische University Eindhoven, October, 1999.

14- Nabritt, S.M., "Modeling Multipath in 802.11 Systems", University of Central Florida, Comms Design, October, 2002.

15- Berenguer, I., "Filtered Multitone (FMT) Modulation for Broadband Fixed Wireless Systems", MSc. Thesis, University of Cambridge, August, 2002.

16- Manishankar, R., "Receiver Performance in IEEE 802.11a Wireless LAN Standard", MSc. Thesis, The Faculty of the Graduate school University of Missouri - Columbia, December, 2002.

17- Siew, J., Piechocki, R., Nix, A. and Armour, S., "A Channel Estimation Method for MIMO - OFDM Systems", Center of Communication Research, University of Bristol, 2002.

18- Barbero, L.G. and Thompson, J.S., "Rapid Prototyping of MIMO Algorithms for OFDM WLAN", Institute for Digital Communications, University of Edinburgh, 2003.

19- Hara, Y., Taira, A., Suto, K. and Ohtsuki, T., "Throughput Enhancement for MIMO - OFDM Systems Using Transmission Control and Adaptive Modulation", Technology Center Europe B.V. (ITE), 2004. 